\title{
Wireless Sensor Network Using Six Sigma Multi Hop Routing
}

\author{
\#Amit Sharma ${ }^{1}$, P. V. Singh ${ }^{2}$, Neelam Srivastava ${ }^{3}$ \\ ${ }^{I}$ Deptt. of E\&C Engg., KIMT, Moradabad, U.P., ${ }^{2}$ Deptt. Of E\&C Engg., KIMT, Moradabad, U.P., ${ }^{3}$ Deptt. of \\ E\&C Engg., IET, Lucknow, \# Author 1 is a research scholar at IFTM University Moradabad, UP. India
}

\begin{abstract}
The most significant problem in the design of wireless sensor networks is to coordinate the sensors with dynamism into a wireless communication network and route sensed data to the base station. The energy efficiency is the most important key point of the network routing designing. This paper presents the efficient multi hop routing algorithm to extend the lifetime of sensor networks and focuses by employing six sigma principles to obtain the Quality of Service. To attain QoS support, we have to find either a route to assure the application requirements or offering network response to the application when the requirements cannot be met.

Keywords: Wireless Sensor Networks, Multi hop routing, six sigma, QoS.
\end{abstract}

\section{Introduction}

Energy competence is a major factor in ad-hoc sensor networks since the nodes are deployed in an infra-structure less environment which demands limited usage of energy. A lot of research works focus on energy competent routing protocols to handle this issue. Currently, the growing interest in multimedia applications has made the Quality of Service (QoS) supporting a necessary task. The Wireless Sensor Networks (WSN) consists of a set of sensors that communicate with each other to accomplish a common task. These huge numbers of nodes, which have the capability to communicate wirelessly, to execute limited computation, and to sense their environment, form the WSN. Most applications for WSNs engage battery- powered nodes with limited energy. When a node exhausts its energy, it cannot sense or relay data any more. Thus, current research on sensor networks mostly focus on protocols with energy efficient mechanisms. Therefore, in the design of WSNs both energy-efficient and QoS supporting issues should be considered in order to guarantee effective field data collection and optimal network energy allocation.

Advances in processor, memory and radio technology will enable small and cheap nodes capable of sensing, communication and computation. Networks of such nodes called wireless sensor networks can of coordinate to perform distributed sensing environmental phenomena.

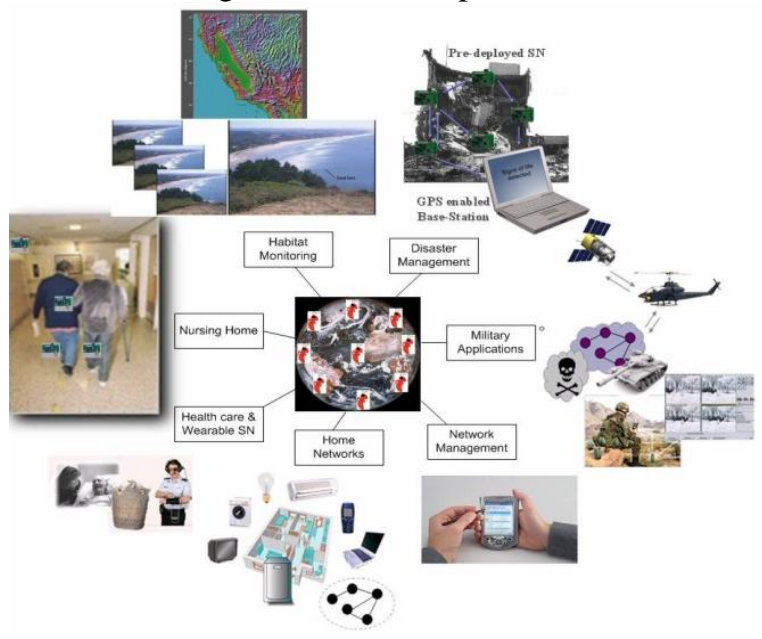

Fig.1.

Networks Sensor networks have emerged as a promising tool for monitoring (and possibly actuating) the physical world, utilizing self-organizing networks of battery- powered wireless sensors that can sense, process and communicate.

\section{RELATED WORK}

The extensive work related to this paper can be categorized into efficient QoS approach in multi hop routing protocol and incorporating six sigma principles. 


\title{
A. Multi Hop Routing
}

In the absence of default router, every node acts as a router and forwards each other's packets to smooth the progress of information sharing between mobile hosts. Multi-hop routing can effectively conquer shadowing and path loss effects to offer exposure over large geographical regions. Through multi-hop communication it is feasible to improve sensing accurateness by distributed processing of large quantities of sensing information. Since the availability of multiple paths for the data to flow obtainable by multi-hop routing, it is easy to maintain sensor network functionalities without any interruption due to sensor node failures $[5,6]$.

\section{B. Quality of Service (QoS)}

In some applications, data should be delivered within a certain period of time from the instant it is sensed; otherwise it becomes useless. In many applications, preservation of energy, which is directly interrelated to network lifetime, is considered comparatively more important than the quality of data sent [4]. As the energy gets exhausted, the network may be required to compromise the quality of the results in order to decrease the energy dissipation in the nodes. Hence, energy aware routing protocols are required to capture this requirement. QoS-based routing protocols have to balance between power utilization and contentment of certain QoS metrics, such as end-to-end delay and bandwidth when delivering information to the base station. QoS aspects include bandwidth constraints due to a generally shared medium, dynamic topology since the topology of mobile nodes may vary and the power expenditure due to inadequate batteries [2]. In a multi-hop wireless sensor network, the QoS requirement like end-to-end bandwidth and delay management firmly depends upon network topology. In the lack of a proper network configuration, some nodes might emerge to be highly error prone, and as a result, it might be difficult to find a QoS path for the function of the network [7].

\section{Challenges for QoS Provisioning for Multi hop Wireless Networks}

In wireless multi-hop networks such as MANETs and WSNs, QoS provisioning is very challenging when compared to wired networks because of a range of difficulties related with these types of networks $[8,9]$.

\section{i) Capacity Constraints:}

In multi-hop wireless networks, wireless bandwidth is inadequate and expensive. They are generally operational with a single radio interface only, restricting the communication capability of these nodes.

\section{ii) Unreliable Communication Medium:}

In multi-hop wireless networks, the wireless medium which is used for communication purposes is prone to errors due to interference generated from transmissions of other wireless devices in the surrounding area, as well as multi-path loss effects. This makes it particularly challenging to offer any guarantees regarding consistent packet delivery.

\section{iii) Inaccurate Bandwidth Estimation:}

It is difficult to exactly determine the accessible wireless channel bandwidth at a router, as it is affected by a number of factors, including the traffic load in the wireless transmission and sensing range, node mobility, as well as the general unpredictability of wireless links.

\section{iv) Lack of Centralized QoS Control:}

There is no centralized controller in a multi-hop wireless networks which is used to keep track of node's location and the remaining QoS resources information of the network. There fore, QoS provisioning has to be done in a distributed manner, which is much more difficult than for a centralized network.

\section{QoS-Aware Routing}

QoS is an agreement to provide assured services, such as bandwidth, delay and packet delivery ratio to users. The Bandwidth evaluation is the key to supporting QoS. This work focuses on exploring ways to calculate approximately the available bandwidth, incorporating a QoS aware scheme into the route discovery process. The available end-to-end bandwidth along a route from the source to the destination must be known in order to propose bandwidth-guaranteed QoS. Here, the bandwidth is shared among neighboring hosts, and an individual host has no knowledge about other neighboring hosts' traffic position [3].

\author{
Algorithm 1: QAR Routing \\ 1. Input: source sends a RREQ (bandwidth request). \\ 2. Output: minimum bandwidth. \\ 3. When intermediate host receives the RREQ packet, it calculates its residual bandwidth.
}


4. If residual bandwidth > minimum bandwidth

Forward RREQ Else Update minimum bandwidth using its residual bandwidth.

5. Compute minimum bandwidth.

6. Destination host sends RREP with a minimum bandwidth to the source host.

The -Hello messages is used by every host to broadcast information about the bandwidth it is currently using and for a host to estimate its available bandwidth based on the bandwidth consumption indicated in the -Hello messages from its two-hop neighbors. The Hello messages provide information about available bandwidth of one-hop neighbors in order to evaluate the amount of free resources on a link [10].

\section{E. six sigma principles on QoS}

Six Sigma is systematically a process of quality improvement through the disciplined data-analysing approach, and by improving the process by eliminating the defects or the obstacles which prevents to reach the perfection. It can be said that the processes that operates with the Six Sigma quality, is able to produce a quality products at a low rate of defects. Six Sigma is employed over wireless sensor networks to ensure QoS guarantees.Six Sigma uses a single set of problem-solving techniques such as DMAIC (Define, Measure, Analyse, Improve and Control). It is used for the purpose of incremental improvements to the existing processes.

\section{The major steps involved in calculating Sigma for any process:}

1. Calculate the mean.

2. Find the deviations from the arithmetic mean

3. Square each deviation.

4. Add the squared deviations.

5. Find the average of the squared deviations, this is known as the variance.

6 . Take the square root of the variance.

This can be expressed mathematically as

$\sigma=\sqrt{ } \sum(\chi-\gamma) 2 / \mathrm{n}$

Where,

$\sigma=$ standard deviation

$\Sigma=$ sum or total.

$\chi=$ individual data.

$\gamma=$ arithmetic mean.

$\mathrm{n}=$ Total no. of observations.

This work is motivated from the research conducted on employing Six Sigma over hybrid sensor networks [1].

\section{System Design}

The objective of Six Sigma quality is to reduce process output variation. Six Sigma seeks to improve the quality of process outputs by identifying and removing the causes of defects and minimizing variability in process.
Algorithm 2: SSMHR
1. Input: $\Sigma, \chi, \gamma, n$
2. Output: $\sigma$
3. If $n<5$ and if $n=1$ then
4. Compute the current rate and new rate
5. If $n>=5$ then
6. Calculate total rate which will be obtained by adding the total rate and current rate.
7. Compute mean rate by dividing the total rate with the total no of observations.
8. By using the values calculate the deviation by applying the equation (1).
9. Then new rate will be the deviation.
Here $\chi$ is current rate, $\gamma$ is mean rate, $\Sigma$ is total rate and $n$ is the number of observations.

The quality improvement will be based on incremental optimization. The main objective of this implementation is to reduce the data transfer time thereby improving the packet delivery ratio and to find settings for the data rate that optimizes the output and minimizing the delay.

As the process sigma value increases from zero to six, the variation of the process around the mean value decreases. With a high enough value of process sigma, the process approaches zero variation and is known as 'zero defects.' 


\section{Simulation And Results}

This work is implemented using the Network Simulator Ns- 2.32. The simulation environment is chosen with the following parameters:

1. Number of nodes : 100

2. Antenna Directional : Omni

3. Network Area : $1500 * 1500 \mathrm{~m}$

4. Traffic (CBR) : 2 Mbps

5. Radio Range : $260 \mathrm{~m}$

6. MAC Layer : IEEE 802.11 CSMA/CD

7. Routing protocol : SSMHR

8. Node Max Speed : $5 \mathrm{~m} / \mathrm{s}$

9. Mobility Model : Random Waypoint

10. Sensor Range : $10-160 \mathrm{~m}$

11. Range of limited Flood : 2 hops

12. Energy (in Joules) : $100 \mathrm{~nJ} / \mathrm{bit}$.

The metrics used for comparison are packet delivery ratio and End -to-End Delay. Packet Delivery Ratio (PDR) is defined as the ratio of the number of data packets received versus the number of data packets sent.

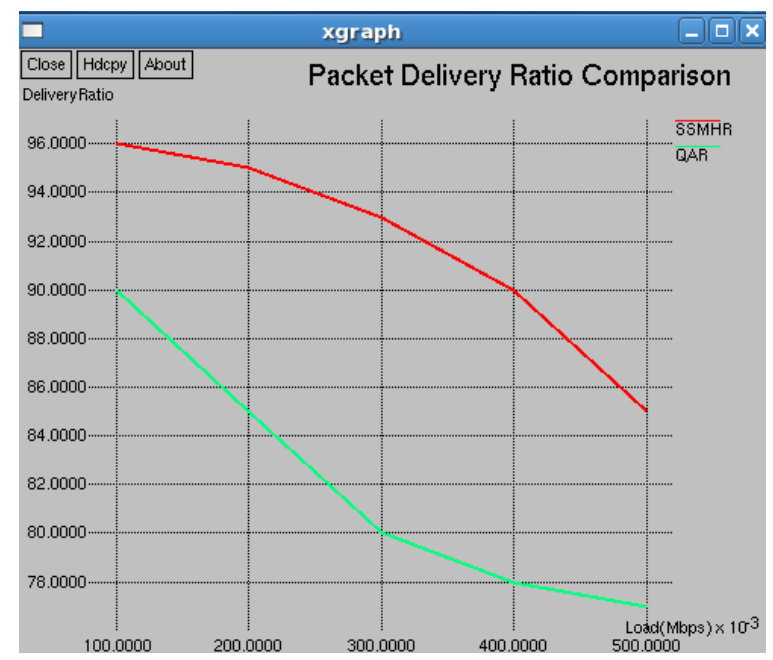

Fig.2. Packet Delivery Ratio Comparison

Fig.2. shows that when compared to QAR, SSMHR increases the packet delivery ratio significantly. End-to-End Delay is the average time from when a data packet is originated by a source until it is successfully received by a receiver. Fig.2.shows that the improvement in packet delivery ratio also impacts reduced delay.

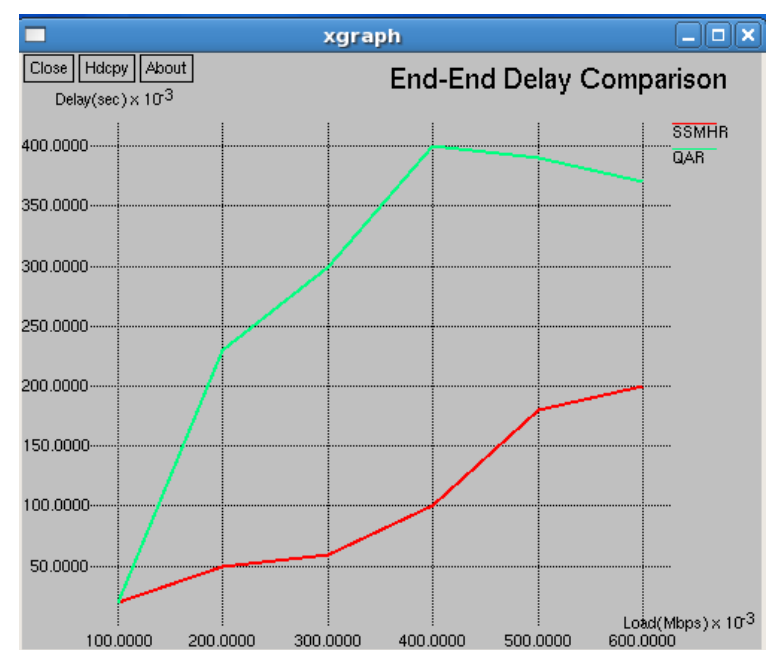

Fig.3. End -End Delay Comparison 


\section{Application}

Wireless Sensor Networks may consist of many different types of sensors such as seismic, low sampling rate magnetic, thermal, visual, infrared, acoustic and radar. They are able to monitor a wide variety of ambient conditions that include temperature, humidity, vehicular movement, lightning condition, pressure, soil makeup, noise levels, the presence or absence of certain kinds of objects, mechanical stress levels on attached objects, and the current characteristics such as speed, direction and size of an object.

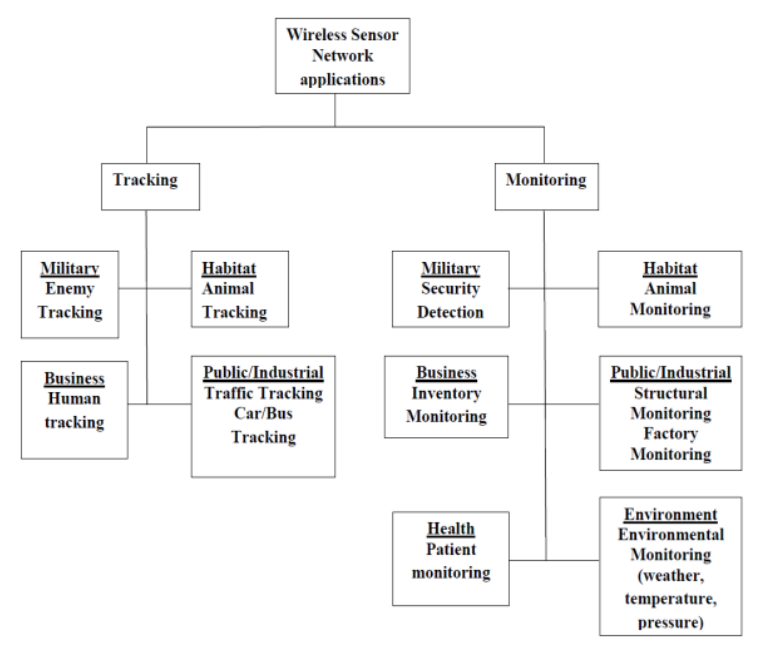

Figure .4: Overview of Wireless Sensor Network applications

\section{CONCLUSION}

This paper implements Six Sigma principles to reduce the variations and measure of quality that strives for near perfection to ensure QoS guarantees in wireless sensor networks. Simulations show that our proposed SSMHR routing protocol can improve packet delivery ratio greatly while also decreasing the End-to-End delay compared to existing $\mathrm{QOS}$ aware routing mechanism. The future work focuses on further experimentation to improve the effectiveness of the proposed approach.

\section{References}

[1] Gandhiyavendhan.S, S.V.Manisekaran, -An analysis to Coverage in Hybrid Mobile Sensor Networks using Six Sigma, International Journal of Advanced Engineering and Application, Issue 01. pp 25-27, January 2010

[2] Du, Keming Yang, Yahui, —A QoS Routing for Maximum Bandwidth in Ad Hoc Networks, Second International Conference on Future Networks, pp 343 - 345, January 2010.

[3] Lei Chen and Wendi B. Heinzelman ,'QoS-Aware Routing Based on Bandwidth Estimation for Mobile Ad Hoc Networks, IEEE journal on selected areas in communications, vol. 23, no. 3, march 2005.

[4] Aniket Malvankar, Ming Yu and Kin K. Leung, A Dynamic clustering and Energy Efficient Routing Technique for Sensor Networks, IEEE transactions on wireless communications, vol.6, no. 8, 2007

[5] Choi, Seong-Yong Kim, Jin-Su Lee, Jung-Hyun Rim, Kee-Wook, REDM: Robust and Energy Efficient Dynamic Routing for a Mobile Sink in a Multi Hop Sensor Network", Second InternationalConference on Communication Software and Networks, pp 178 -182 , February 2010.

[6] Jian Wen, Min Yu ,Wen-Wen Huang and Ya-Li Peng - Energy- Efficient Multi-hop Hierarchical Routing Protocol for Wireless Sensor Networks , International Conference on Networks Security, Wireless Communications and Trusted Computing, pp 469 472, 2009.

[7] Binod Kumar Pattanayak, Manoj Kumar Mishra Alok Kumar Jagadev, Dr. Manoj Ranjan Nayak, A Cluster-based QoS Support To Bandwidth Preservation With Concept Of Survivability In Multi-hop Mobile Ad Hoc Networks, Proceedings of the World Congress on Engineering and Computer Science, Vol. 1, October 2009.

[8] Bo Rong, Yi Qian, Kejie Lu, and Rose Qingyang Hu, Enhanced QoS Multicast Routing in Wireless Mesh Networks,IEEE Transactions on wireless communications, vol. 7, no. 6, june 2008.

[9] Aisha-Hassan A. Hashim, Mohammad M. Qabajeh, Othman Khalifa and Liana Qabajeh, Review of Multicast QoS Routing Protocols forMobile Ad Hoc Networks, International Journal of Computer Science and Network Security, VOL.8 No.12, December 2008.

[10] Nur Idawati Md Enzai , Farhat Anwar and Zeldi Suryady ,-Analyzing the Effect of HELLO Messages in QoS-AODV, proceedings of the international conference on Electrical engineering and informatics institute teknologi bandung, Indonesia june 17-19,2007. 